\title{
SUSY, Casimir scaling, and probabilistic properties of gluon and quark-jet evolution
}

\author{
Anatoly V. Kotikov and Oleg V. Teryaev (D) \\ Joint Institute for Nuclear Research, 141980 Dubna, Russia
}

(Received 28 January 2020; revised 25 November 2020; accepted 4 January 2021; published 3 February 2021)

\begin{abstract}
We study the new relation [B. A. Kniehl and A. V. Kotikov, arXiv:1702.03193.] between the anomalous dimensions, resummed through next-to-next-to-leading logarithmic order, in the Dokshitzer-GribovLipatov-Altarelli-Parisi evolution equations for the first Mellin moments $D_{q, g}\left(\mu^{2}\right)$ of the fragmentation functions, which correspond to the average multiplicities of hadrons in jets initiated by quarks and gluons, respectively. This relation is shown to lead to probabilistic properties of the properly rescaled parton jet multiplicities obtained from standard ones by extracting the quark and gluon "color charges" $C_{F}$ and $C_{A}$, respectively.
\end{abstract}

DOI: $10.1103 /$ PhysRevD.103.034002

\section{INTRODUCTION}

The broad and elegant concept of supersymmetry (SUSY) is currently manifested in various branches of physics. For high energies, it is pronounced in the properties of QCD supersymmetric extension rather than in the existence of supersymmetric partners. In particular, this corresponds to the SUSY-related properties of evolution kernels [1] discovered some time ago [2]. In the current paper, we explore the recently found relation [3] for fragmentation kernels and suggest its probabiolistic interpretation, bringing SUSY closer to observations.

The notion of fragmentation functions (FFs) $D_{a}\left(x, \mu^{2}\right)$ [hereafter, $(a=q, g)$ ], where $\mu$ is the factorization scale, was involved during the study of the inclusive production of single hadrons. Their $\mu^{2}$ dependence is governed by the timelike Dokshitzer-Gribov-Lipatov-Altarelli-Parisi (DGLAP) evolution equations [1,2],

$$
\mu^{2} \frac{\partial D_{a}\left(x, \mu^{2}\right)}{\partial \mu^{2}}=\sum_{b} P_{a b}(x) \otimes D_{b}\left(x, \mu^{2}\right),
$$

where $P_{a b}(x)$ are the timelike splitting functions, and the symbol $\otimes$ marks the Mellin convolution,

$$
f_{1}(x) \otimes f_{2}(x) \equiv \int_{x}^{1} \frac{d z}{z} f_{1}\left(\frac{x}{z}\right) f_{2}(z)
$$

Published by the American Physical Society under the terms of the Creative Commons Attribution 4.0 International license. Further distribution of this work must maintain attribution to the author(s) and the published article's title, journal citation, and DOI. Funded by SCOAP ${ }^{3}$.
The DGLAP equations are conveniently solved in Mellin space (hereafter, we represent the Mellin moment $N$ as $N=1+\omega)$,

$$
\mu^{2} \frac{\partial D_{a}\left(\omega, \mu^{2}\right)}{\partial \mu^{2}}=\sum_{b} P_{a b}(\omega) D_{b}\left(\omega, \mu^{2}\right)
$$

where

$$
D_{a}\left(\omega, \mu^{2}\right)=\int_{0}^{1} d x x^{\omega} D_{a}\left(x, \mu^{2}\right)
$$

are FF Mellin moments. Here, $P_{a b}\left(N, a_{s}\right)$ [hereafter, $(a, b=q, g)]$ are anomalous dimensions [i.e., the Mellin moments of the corresponding splitting functions $\left.P_{a b}\left(x, a_{s}\right)\right]$,

$$
P_{a b}\left(\omega, \mu^{2}\right)=\int_{0}^{1} d x x^{\omega} P_{a b}\left(x, \mu^{2}\right)
$$

and $D_{s}=\left(1 / 2 n_{f}\right) \sum_{q=1}^{n_{f}}\left(D_{q}+D_{\bar{q}}\right)$, with $n_{f}$ being the number of active quark flavors, is the quark singlet component. The quark nonsinglet component is irrelevant for the present study.

The timelike splitting functions $P_{a b}\left(x, a_{s}\right)$ and the corresponding anomalous dimensions $P_{a b}\left(\omega, a_{s}\right)$ in Eq. (1.3) may be computed perturbatively in $a_{s}$,

$$
\begin{gathered}
P_{a b}\left(x, a_{s}\right)=\sum_{k=0}^{\infty} a_{s}^{k+1} P_{a b}^{(k)}(x), \\
P_{a b}\left(\omega, a_{s}\right)=\sum_{k=0}^{\infty} a_{s}^{k+1} P_{a b}^{(k)}(\omega),
\end{gathered}
$$


where $a_{s}\left(\mu^{2}\right)=\alpha_{s}(\mu) /(4 \pi)$ is the coupland. The functions $P_{a b}^{(k)}(x)$ and $P_{a b}^{(k)}(\omega)$ for $k=0,1,2$ in the $\overline{\mathrm{MS}}$ scheme may be found in Refs. [4-6] through the next-to-next-to-leading order (NNLO).

The first Mellin moment $D_{a}\left(\mu^{2}\right) \equiv D_{a}\left(1, \mu^{2}\right)$ is of special interest. Up to corrections of orders beyond our consideration here, this corresponds to the average multiplicity $\left\langle n_{h}\right\rangle_{a}$ of hadrons in the jets initiated by parton $a$. Now, there are a lot of experimental data on $\left\langle n_{h}\right\rangle_{q},\left\langle n_{h}\right\rangle_{g}$, and their ratio $r=\left\langle n_{h}\right\rangle_{g} /\left\langle n_{h}\right\rangle_{q}$ for charged hadrons $h$ taken in $e^{+} e^{-}$annihilation at different energies $\sqrt{s}$ of the center of mass, ranging from 10 to $209 \mathrm{GeV}$ (see a list of references in [7]). The study of $D_{a}$ contains a long story: The leading order (LO) value of $r, C^{-1}=C_{A} / C_{F}$ with color factors $C_{F}=4 / 3$ and $C_{A}=3$, was found four decades ago [8]. ${ }^{1}$

Usage of Eq. (1.3) with $N=1$ for $D_{a}$ at fixed order in perturbation theory is problematic: $P_{a b} \equiv P_{a b}(N=1)$ are ill defined and require resummation, which was performed for the leading logarithms (LL) [18], the next-to-leading logarithms (NLL) [19], and the next-to-next-to-leading logarithms (NNLL) [20].

In Ref. [3] (see also [21]), an unexpected relation between the NNLL-resummed expressions for $P_{b a}$ has been found. Its existence in QCD is quite remarkable and interesting in its own right, because a similar relationship is familiar $[2,20,22]$ from supersymmetric QCD (SQCD), where $C=1$.

In the present paper, we will show that the relation obtained in Ref. [3] leads to probabilistic properties of the rescaled average multiplicities.

The paper is organized as follows. In Sec. II, we consider the basic properties and the results of the resummation for the anomalous dimensions of the fragmentation functions. The results are given by standard procedure [18], extended to the $\overline{\mathrm{MS}}$ scheme in Ref. [23], as well by Vogt approach $[19,20]$. In Sec. III, we present the two different procedures of diagonalization. In the first one, we diagonalize the (LO of) the DGLAP equation for arbitrary $N$ values and later take the limit $\omega \rightarrow 0$ for $N=1+\omega$. Such diagonalization is also useful to study the full FF evolution. In the second possibility, we consider directly the first moment $N=1$ and diagonalize the gluon and quark multiplicities themselves. Section IV contains discussions of Casimir scaling and probabilistic properties of gluon and quark jet evolution.

\footnotetext{
${ }^{1}$ One should stress that the multiplicities $D_{a}\left(\mu^{2}\right)$ obey to socalled "Casimir scaling," since their results are given by universal function times the quadratic Casimir operators, i.e., to $C_{F}$ and $C_{A}$ for the fundamental and adjoint representations [9] of the color SU(3) group, respectively. (See Refs. [10-16] and discussions therein about the Casimir scaling, which appeared in the 1980s [17] in lattice calculations).
}

\section{RESUMMATION}

To explicate the ideas of resummation, we consider here the cross section for the semi-inclusive hadron production in electron-positron annihilation:

$$
e^{+}\left(k_{1}\right)+e^{-}\left(k_{2}\right) \rightarrow V^{*}(q) \rightarrow h\left(p_{h}\right)+X,
$$

where $V^{*}$ is a virtual vector boson with virtuality $Q^{2}=q^{2}=$ $\left(k_{1}+k_{2}\right)^{2}$, and $X$ stands for any allowed hadronic final state. Here, we are interested in the differential cross section for the single hadron production $d \sigma^{h}\left(x, Q^{2}\right) / d x$ [or the structure function $F\left(x, Q^{2}\right)=\left(d \sigma^{h}\left(x, Q^{2}\right) / d x\right) / \sigma_{k}^{(\mathrm{ew})}$, where $\sigma^{(\mathrm{EW})}$ contains all the electroweak overall factors], where $x$ is the scaled momentum fraction of the produced hadron $h$ :

$$
x=\frac{2 p_{h} \cdot q}{Q^{2}}, \quad 0 \leq x \leq 1 .
$$

According to QCD factorization the structure function, $F\left(x, Q^{2}\right)$ can be written as a convolution of the coefficient function $C_{a}\left(x, Q^{2}\right)$ (i.e., the parton cross section to produce a parton $a$, divided by factor $\sigma^{(\mathrm{EW})}$ ) with the fragmentation function $D_{a}(x)$ from the parton:

$$
F\left(x, Q^{2}\right)=\sum_{a} C_{a}\left(x, Q^{2}\right) \otimes D_{a}(x),
$$

where the symbol $\otimes$ is defined in Eq. (1.2).

As we already discussed in the Introduction, perturbation theory does not work properly when the fraction $x$ of available energy carried away by the observed particle is too small, since large logarithms spoil the convergence of the perturbation theory series. The largest logarithms, double logarithms (DLs) contribute to the splitting functions $P_{a, b}\left(x, a_{s}\right)$, which determine the evolution of the fragmentation function, and were computed for all orders long ago [18]. The total DL contribution to the parton cross sections was calculated in the work [24] for the case where collinear singularities are regularized by imparting a small mass $m_{g}$ to the gluon, in the so-called massive gluon (MG) regularization scheme. As noted in [23] pioneering the NNLO approximation, the results found in Ref. [24] do not match the results computed at a fixed order (see Refs. [25-27]), which is not surprising, because the two computations were performed in two different regularization and factorization schemes, namely the MG and the $\overline{\mathrm{MS}}$ scheme. DL gluon coefficients were calculated in the $\overline{\mathrm{MS}}$ scheme in [23]. In the Subsection II A, we present the result in a less formal but simpler way.

So, below we demonstrate the basic ideas of the resummation of the fragmentation functions, which is important near its first Mellin moment. We consider an extension to the $\overline{\mathrm{MS}}$ scheme [23] (see also a short review in 
Ref. [28]) of the traditional approach $[18,24]$ of resummation (see Sec. II A) and also show the important points of the Vogt et al. approach $[19,20]$, which gives a possibility to have an accurate treatment of the resummation up to NNLL level of accuracy.

\section{A. Traditional approach to resummation}

It is well known that these DL contributions appear in the gluon-gluon and gluon-quark timelike splitting functions [18] and in the timelike gluon coefficient function [24].

To extract them, consider a general process with a final singlet color state, including the creation of an "observed" gluon with momentum $q$ from a hard parton with momentum $p$, around which, a jet is formed. In the DLA, the DL contributions arise from unobservable soft gluons in the final state. Therefore, there must be an additional hard parton to take into account the recoil from the parton of momentum $p$ as a result of momentum conservation. The momentum of this extra parton is written as $\bar{p}$. The cross section for this process will be written $d \sigma(p, \bar{p}, q)$. A typical example is the process $e^{+}+e^{-} \rightarrow V^{*} \rightarrow$ $\mathcal{Q}(p)+\overline{\mathcal{Q}}(\bar{p})+g(q)+X$, where $V^{*}=\gamma, Z$ is the virtual vector boson, where the jet is formed around the quark $\mathcal{Q}$ with momentum $p$ and around the antiquark $\overline{\mathcal{Q}}$ with momentum $\bar{p}$, and where $X$ is any hadronic final state allowed by the conservation of the quantum number. Thus, in order to obtain the DL contribution to the cross section, we consider a configuration in which the unobservable part consists only of $N$ soft gluons of momenta $q_{1}, q_{2}, \ldots, q_{N}$, whose phase space is fully integrated. Therefore, defining $d \sigma_{N}\left(p, \bar{p}, q_{1}, q_{2}, \ldots, q_{N}\right)$ as a section in which $N$ gluon momenta $q_{\alpha}, \alpha=1,2, \ldots, N$, are produced together with $p$ and $\bar{p}$ momentum partons, we can write

$d \sigma(p, \bar{p}, q)=\sum_{N=0}^{\infty} d \sigma_{N+1}\left(p, \bar{p}, q, q_{1}, q_{2}, \ldots, q_{N}\right)$,

where it is understood that the $q_{\alpha}$ are fully integrated over, but not $q$. It is a well-known result [29] that the DL contributions come from the kinematic configuration in which the momenta of the soft gluons and also the angles $\theta_{i}$ of the emitted gluons with respect to the hard parton of momentum $p$ are strongly ordered; i.e.,

$$
\begin{aligned}
|\vec{q}| & \ll\left|\vec{q}_{1}\right| \ll\left|\vec{q}_{2}\right| \ll \cdots \ll\left|\overrightarrow{q_{N}}\right| \ll Q / 2, \\
\theta & \ll \theta_{1} \ll \theta_{2} \ll \cdots \ll \theta_{N} \ll 1,
\end{aligned}
$$

where $\theta$ refers to the gluon of momentum $q$.

To extract the LL behavior, we use the single-gluon probability emission factorization in the soft collinear limit. This is a consequence of the eikonal approximation and color coherence, as has been proven long ago in $[29,30]$. This result was used [23] to obtain the probability of gluon emission in $d=4-2 \epsilon$ dimensions:

$$
d w(x, z, \epsilon)=2 C_{i} a_{s}\left(\frac{\mu^{2}}{Q^{2}}\right)^{\epsilon} \frac{(4 \pi)^{\epsilon}}{\Gamma(1-\epsilon)} \frac{d x}{x^{1+2 \epsilon}} \frac{d z}{z^{1+\epsilon}},
$$

where $z=(1-\cos \theta) / 2$, with $\theta$, the scattering angles of the emitted soft gluon with respect to the hard jet direction. Here, $C_{i}=C_{A}$ for a gluon jet, and $C_{i}=C_{F}$ for a quark jet. The expression for the probability emission given in Eq. (2.6) is what we need to obtain the gluon probability density in dimensional regularization.

The consistency relation for the differential cross section for the gluon jet production has the following form:

$$
d \sigma_{g}^{n}=d \sigma_{g}^{1}+d \sigma_{g}^{n-1} d w(x, z, \epsilon)
$$

Taking the limit $n \rightarrow \infty$, we obtain immediately the following bootstrap equation:

$$
\begin{aligned}
x^{1+2 \epsilon} \mathcal{G}(x, z, \epsilon)= & \delta(1-x)+\int_{x}^{1} d x^{\prime} \\
& \times \int_{z}^{1} d z^{\prime} K\left(x^{\prime}, z^{\prime}, \epsilon\right) x^{\prime 1+2 \epsilon} \mathcal{G}\left(x^{\prime}, z^{\prime}, \epsilon\right),
\end{aligned}
$$

where $K(x, z, \epsilon)=d w(x, z, \epsilon) / d x d z$ and $\mathcal{G}(x, z, \epsilon)$ is an angle-dependent gluon density. The factor of $x^{1+2 \epsilon}$ represents our normalization coming from the explicit computation for the first gluon emission with $n=2$.

Now, we introduce the gluon distribution density $G(x, \epsilon)$ as $\mathcal{G}(x, z=0, \epsilon)=G(x, \epsilon)$. Let us perform the Mellin transform, of Eq. (2.8) as well as in Eqs. (1.4) and (1.5) above. Further, integrating over $z$, solving recursively the equation for $\mathcal{G}(x, z, \epsilon)$ and finally putting $z=0$, we obtain (see details in Ref. [23]),

$$
\begin{aligned}
G(\omega, \epsilon) & =1+\sum_{k=1}^{\infty}\left(\frac{X}{2 \varepsilon^{2}}\right)^{k} \frac{\Gamma(1+\nu)}{k ! \Gamma(k+1+\nu)} \\
& =\Gamma(1+\nu)\left(\frac{Z}{2}\right)^{\nu} I_{\nu}(Z),
\end{aligned}
$$

where

$$
X=2 C_{A} a_{s} \frac{(4 \pi)^{\epsilon}}{\Gamma(1-\epsilon)}\left(\frac{\mu^{2}}{Q^{2}}\right)^{\epsilon}=2 C_{A} a_{s}+O(\varepsilon)
$$

and $\nu=-\omega /(2 \epsilon)$ and $z=\sqrt{2 A} / \varepsilon$ and $I_{\nu}(z)$ is modified Bessel function.

According to the QCD factorization theorem, all collinear singularities in Eq. (2.9) should be factorized. In the $\overline{\mathrm{MS}}$ factorization scheme, this requires comparing Eq. (2.9) with (see [31,32]), 


$$
\begin{aligned}
G(\omega, \epsilon)= & C^{\overline{\mathrm{MS}}}\left(\omega, a_{s}, \frac{Q^{2}}{\mu_{F}^{2}}\right) \\
& \times \exp \left[-\frac{1}{\epsilon} \int_{0}^{a_{s}\left(\mu_{F}^{2}\right)} \frac{d a}{a} P^{\overline{\mathrm{MS}}}(\omega, a)\right],
\end{aligned}
$$

to the proper accuracy.

The direct comparison of Eqs. (2.9) and (2.11) is highly nontrivial. However, it is well known that Bessel function obeys the second order differential equations. Indeed, from Eq. (2.9), it is easy to check that $G$ satisfies the following simple differential equation:

$$
\frac{\ddot{G}-\frac{\omega}{2 \epsilon} \dot{G}}{G}=\frac{X}{2 \epsilon^{2}}, \quad \dot{f}(X) \equiv X \frac{d f}{d X}=a_{s} \frac{d f}{d a_{s}} .
$$

From Eq. (2.11), we have

$$
\frac{\dot{G}}{G}=\frac{\dot{C}}{C}-\frac{P}{\epsilon}, \quad \frac{\ddot{G}}{G}=\frac{\ddot{C}}{C}-2 \frac{P}{\epsilon} \frac{\dot{C}}{C}-\frac{\dot{P}}{\epsilon}+\frac{P^{2}}{\epsilon^{2}},
$$

so that

$$
\frac{\ddot{G}-\frac{\omega}{2 \epsilon} \dot{G}}{G}=\frac{1}{\epsilon^{2}}\left(P^{2}+\frac{\omega P}{2}\right)-\frac{1}{\epsilon}\left(\left[2 \gamma+\frac{\omega}{2}\right] \frac{\dot{C}}{C}+\dot{P}\right)+\frac{\ddot{C}}{C} .
$$

Now, comparing the coefficients of $\epsilon^{-2}$ and $\epsilon^{-1}$ on the right hand side of Eq. (2.14) with those of Eq. (2.12) and noting that $\gamma$ is explicitly independent of $\epsilon$ one gets, respectively,

$$
P^{2}+\frac{\omega P}{2}-\frac{X}{2}=0, \quad \frac{\partial \ln C}{\partial P}=-\frac{2}{4 P+\omega},
$$

and, thus, we have

$$
\begin{aligned}
P\left(\omega, a_{s}\right) & =\frac{1}{4}\left[\sqrt{\left.\omega^{2}+32 C_{A} a_{s}\right]}-\omega\right] \\
& =\frac{1}{4}\left[\sqrt{\omega^{2}+16 \gamma_{0}^{2}}-\omega\right], \\
C\left(\omega, a_{s}\right) & =\left[\frac{\omega}{4 P\left(\omega, a_{s}\right)+\omega}\right]^{\frac{1}{2}},
\end{aligned}
$$

with

$$
\gamma_{0}=\sqrt{2 C_{A} a_{s}}
$$

So, after the resummation, we have a flat limit at $\omega \rightarrow 0$ and

$P\left(\omega=0, a_{s}\right)=\gamma_{0}, \quad C\left(\omega, a_{s}\right)=\left[\frac{\omega}{4 \gamma_{0}+\omega}\right]^{\frac{1}{2}}$,

and $\gamma_{0}$ appears as a new parameter of expansion.

\section{B. Vogt approach}

Here, we show the basic properties of A. Vogt approach [19], where according to all order factorization, the singularities of the splitting functions are iteratively extracted into the transition function $Z(\epsilon)$ of the $\epsilon=0$ poles in dimensional regularization $(d=4-2 \epsilon)$.

According to the factorization theorem [31,33], we can rewrite the expression (2.3) for the structure function $F\left(x, Q^{2}\right)$ in Mellin space $^{2}$ as

$$
\begin{aligned}
F(\omega) & =\sum_{a} C_{a}(\omega) D_{a}(\omega) \\
& =\sum_{a, b, c} \hat{C}_{a}^{0}(\omega, \epsilon) Z_{a b}^{-1}(\omega, \epsilon) Z_{b c}(\omega, \epsilon) \hat{D}_{c}^{0}(\omega),
\end{aligned}
$$

where $\hat{C}_{a}^{0}$ and $\hat{D}_{c}^{0}$ are the so-called "bare" coefficient function and fragmentation function. $Z_{a b}(\epsilon)$ is the transition function containing only poles in $\epsilon$ that are factored out from $\hat{C}_{a}^{0}$. Hence, substituting Eq. (2.19) into Eq. (1.1) at $\mu^{2}=Q^{2}$, we find that the splitting functions can be directly related to the transition function in the following way:

$$
\begin{aligned}
P_{a b}(\omega) & =\sum_{c} Q^{2} \frac{\partial Z_{a c}(\omega)}{\partial Q^{2}} Z_{c d}^{-1}(\omega) \\
& =\beta_{\mathrm{D}}\left(\alpha_{s}\right) \sum_{c} \frac{\partial Z_{a c}(\omega)}{\partial \alpha_{s}} Z_{c b}^{-1}(\omega),
\end{aligned}
$$

where

$$
\beta_{\mathrm{D}}\left(\alpha_{s}\right)=\varepsilon a_{s}-\sum_{i=0} \beta_{i} a_{s}^{i+1},
$$

and $\beta_{0}$ and $\beta_{1}$ values are given below in Eq. (3.16).

As it was shown in Ref. [19], one can solve the Eq. (2.20) in $Z_{a b}$ obtaining at all orders the three highest order poles in $\epsilon$ knowing the NNLO corrections to $P_{a b}$ and $\beta_{i}$. Additionally, knowing the higher order corrections to $C_{a}$, which is pole free, one obtains from the NNLO computations the all order structure of the three first highest singularities in $\epsilon$ :

$$
\hat{C}_{a}^{0}(\omega, \epsilon)=\sum_{b} C_{b}(\omega, \epsilon) Z_{b a}(\omega, \epsilon) .
$$

$\hat{C}_{a}^{0}(\omega, \epsilon)$ may be computed perturbatively in $a_{s}$,

$$
\hat{C}_{a}^{0}(\omega, \epsilon)=\sum_{n} \alpha_{s}^{n} \hat{C}_{a}^{(n)}(\omega, \epsilon),
$$

(and $\quad \hat{C}_{a}^{0}(x, \epsilon)=\sum_{n} \alpha_{s}^{n} \hat{C}_{a}^{(n)}(x, \epsilon)$ in $x$ space $)$.

\footnotetext{
${ }^{2}$ In the $e^{+} e^{-}$process, there are several structure functions, but we consider only $F_{A}$. A consideration of other functions is similar and can be found in Ref. [19].
} 
The key point of Ref. [19] is that, for example, for the case of the gluon, ${ }^{3}$ the small $\omega$ behavior of the bare coefficient functions $\hat{C}_{g}^{(n)}(x, \epsilon)$,

$$
\begin{aligned}
\hat{C}_{g}^{(n)}(x, \varepsilon)= & \frac{1}{\varepsilon^{2 n-1}} \sum_{\ell=0}^{n-1} x^{-1-2(n-\ell) \varepsilon}\left(A_{g}^{(\ell, n)}+\varepsilon B_{g}^{(\ell, n)}\right. \\
& \left.+\epsilon^{2} C_{g}^{(\ell, n)}+\ldots\right)
\end{aligned}
$$

up to nonsingular in $x$ contributions.

Focusing for a moment on the leading logarithms, Eq. (2.24) provides decomposition of $\hat{C}_{g}^{(n)}$, which includes terms of the form $x^{-1} \ln ^{n+m-1} x$ at all orders $\varepsilon^{-n+m}$ with $m=0,1,2, \ldots$, into $n$ contributions of the form,

$$
\varepsilon^{-2 n+1} x^{-1-k \varepsilon}=\varepsilon^{-2 n+1} x^{-1}\left[1-k \varepsilon \ln x+\frac{1}{2}(k \varepsilon)^{2} \ln ^{2} x+\ldots\right],
$$

with $k=2,4, \ldots, 2 n$. Since $\hat{C}_{g}^{(n)}$ starts only at the order $\varepsilon^{-n}$, the coefficients $A_{g}^{(\ell, n)}$ in Eq. (2.24) have to be such that the coefficients of $\varepsilon^{0}, \ldots, \varepsilon^{n-2}$ in the square bracket in Eq. (2.25) cancel the sum of these $n$ contributions. Together, with the three nonvanishing coefficients of $\varepsilon^{-n+\ell}, \ell=0,1,2$, in $\hat{C}_{g}^{(n)}$ known from the above NNLO results, the author of [19] thus had an overconstrained system of $n+2$ linear equations for the $n$ coefficients $A_{g}^{(\ell, n)}$ at each order $n$ of the strong coupling. Similar results have been obtained also for the coefficients $B_{g}^{(\ell, n)}$ and $C_{g}^{(\ell, n)}$.

In the Mellin space, Eq. (2.24) transforms to

$$
\begin{aligned}
\hat{C}_{g}^{(n)}(\omega, \epsilon)= & \frac{1}{\epsilon^{2 n-1}} \sum_{l=0}^{n-1} \frac{1}{\omega-2(n-l) \epsilon}\left(A_{g}^{(l, n)}+\epsilon B_{g}^{(l, n)}\right. \\
& \left.+\epsilon^{2} C_{g}^{(l, n)}+\ldots\right) .
\end{aligned}
$$

Finally comparing Eq. (2.26) with Eq. (2.22), one gets the systems of equations for the coefficients $A_{g}, B_{g}$, and $C_{g}$, which produce sequences up to arbitrary orders in $\alpha_{s}$ of the three highest powers in $1 / \omega$ or equivalently (back to $x$ space) in $\ln x$.

Here, we present the resummed timelike LL and NLL splitting functions obtained in Refs. [19,20],

$P_{a b}(\omega):=\sum_{n=0}^{\infty} a_{\mathrm{s}}^{n+1}\left(\delta_{\mathrm{ag}} P_{\mathrm{ab}, \mathrm{LL}}(\omega)+P_{\mathrm{ab}, \mathrm{NLL}}(\omega)+\ldots\right)$,

where $\delta_{\mathrm{ag}}$ is a Kronecker symbol.

\footnotetext{
${ }^{3}$ The quark function $\hat{C}_{q}^{(n)}(x, \epsilon)$ can be analyzed similarly (see Ref. [19]).
}

The LL and NLL contributions for $P_{\mathrm{gg}}$ have the form,

$$
\begin{gathered}
P_{\mathrm{gg}, \mathrm{LL}}(\omega):=-\frac{\left(-8 C_{A}\right)^{n+1}}{2 \omega^{2 n+1}} A_{\mathrm{gg}}^{(n)}, \\
P_{\mathrm{gg}, \mathrm{NLL}}^{(n) T}(N)=-\frac{(-8)^{n} C_{A}^{n-1}}{3 \omega^{2 n}}\left[\left(11 C_{A}^{2}+2 C_{A} n_{f}\right) B_{\mathrm{gg}, 1}^{(n)}\right. \\
\left.-2 C_{F} n_{f} B_{\mathrm{gg}, 2}^{(n)}\right] .
\end{gathered}
$$

The coefficients in Eq. (2.28) have been determined to order $\alpha_{s}^{16}$, and that leads to the following analytic results:

$A_{\mathrm{gg}}^{(n)}=\frac{(2 n) !}{n !(n+1) !}=\frac{1}{n+1}\left(\begin{array}{c}2 n \\ n\end{array}\right) ; \quad B_{\mathrm{gg}, 1}^{(n)}=\left(\begin{array}{c}2 n-1 \\ n\end{array}\right)$.

The results (2.30) lead to the closed NLL expressions,

$$
\begin{aligned}
\left.P_{\mathrm{gg}}(\omega)\right|_{C_{F}=0}= & \frac{1}{4}\left[\sqrt{\omega^{2}+16 \gamma_{0}^{2}}-\omega\right]-a_{\mathrm{s}}\left(\frac{11}{6} C_{A}+\frac{1}{3} n_{f}\right) \\
& \times\left[\frac{\omega}{\sqrt{\omega^{2}+16 \gamma_{0}^{2}}}+1\right]+\cdots
\end{aligned}
$$

with $\gamma_{0}$ defined in Eq. (2.18).

We see the full agreement of the LL part with $P\left(\omega, a_{s}\right)$ in Eq. (2.16). To obtain more complicated NNLL results, the authors of $[19,28]$ used the results $(2.30)$ as an initial form to present an ansatz for the corresponding NNLL results (see [34] and discussions therein). ${ }^{4}$

\section{Results for multiplicities}

The NLL and NNLL results for $P_{a b}(\omega)$ are rather cumbersome and can be found in Refs. [19,23]. So, here, we present only the results for multiplicities, i.e., for $P_{a b}(\omega=0) \equiv P_{a b}$.

Consider now Eq. (1.3) for $\omega=0$ with NNLL resummation, where [20]

$$
\begin{aligned}
& P_{a a}=\gamma_{0}\left(\delta_{a g}+K_{a}^{(1)} \gamma_{0}+K_{a}^{(2)} \gamma_{0}^{2}\right), \\
& P_{g q}=C\left(P_{g g}+A\right), \quad P_{q g}=C^{-1}\left(P_{q q}+A\right),
\end{aligned}
$$

with $\mathcal{O}\left(\gamma_{0}^{3}\right)$ accuracy, where $\gamma_{0}$ is given in Eq. (2.18), $\delta_{a b}$ is the Kronecker symbol, and

\footnotetext{
${ }^{4}$ Similar ideas have been used also to find [35] an ansatz for $\varepsilon$ expansion coefficients in (series representations) of massive diagrams and also to generate [36,37] an ansatz in calculations in the framework of $\mathcal{N}=4$ super Yang-Mills (SYM) theory. In particular, such an approach gives a possibility to find a so-called universal anomalous dimension in $\mathcal{N}=4 \mathrm{SYM}$ up to seven loops (see [38] and references therein).
} 


$$
\begin{aligned}
K_{q}^{(1)}= & \frac{2}{3} C \varphi, \quad K_{g}^{(1)}=-\frac{1}{12}[11+2 \varphi(1+6 C)], \\
K_{q}^{(2)}= & -\frac{1}{6} C \varphi[17-2 \varphi(1-2 C)], \\
K_{g}^{(2)}= & \frac{1193}{288}-2 \zeta(2)-\frac{5 \varphi}{72}(7-38 C) \\
& +\frac{\varphi^{2}}{72}(1-2 C)(1-18 C), \quad A=K_{q}^{(1)} \gamma_{0}^{2},
\end{aligned}
$$

with $C=C_{F} / C_{A}$ introduced above and

$$
\varphi=\frac{n_{f}}{C_{A}} .
$$

Equation (2.32) is written in a form that allows us to glean a novel relation (see [3]):

$$
P_{q q}+C^{-1} P_{g q}=P_{g g}+C P_{q g},
$$

which is independent of $n_{f}$.

Note that the form of Eq. (2.32), as well as Eq. (2.35) itself, was obtained in [3] by diagonalizing the quark and gluon multiplicities (see Subsection III A). Quite unexpectedly, in the results for diagonal anomalous dimensions (3.9), all square roots were canceled, and the equations (3.14) were obtained. Such root cancellation was previously observed only in supersymmetric generalizations of QCD. The reason for this simplification is associated with the resummation of the anomalous differences and with the direct diagonalization of parton multiplicities done in Subsection III A, which leads to some kind of symmetry. It is very important to look at its possible violation in higher orders of the perturbation theory and in the resummation of higher logarithms. This is a very difficult problem requiring further investigation.

\section{DIAGONALIZATION}

In the general case, it is impossible to diagonalize Eq. (1.3), since the contributions to the matrix of the timelike splitting functions do not commute in different orders. The usual approach is to write a series expansion for the LO solution, which, in turn, can be diagonalized. Thus, we start by choosing a basis in which the matrix of the timelike LO splitting function is diagonal (see, e.g., Ref. [39]),

$$
\begin{array}{ll}
U_{\omega}^{-1}\left(\begin{array}{ll}
P_{q q}^{(0)}(\omega) & P_{g q}^{(0)}(\omega) \\
P_{q g}^{(0)}(\omega) & P_{g g}^{(0)}(\omega)
\end{array}\right) & U_{\omega}=\left(\begin{array}{ll}
P_{--}^{(0)}(\omega) & 0 \\
0 & P_{++}^{(0)}(\omega)
\end{array}\right), \\
U_{\omega}^{-1}\left(\begin{array}{ll}
P_{q q}^{(k)}(\omega) & P_{g q}^{(k)}(\omega) \\
P_{q g}^{(k)}(\omega) & P_{g g}^{(k)}(\omega)
\end{array}\right) & \left.U_{\omega}=\left(\begin{array}{ll}
P_{--}^{(k)}(\omega) & P_{+-}^{(k)}(\omega) \\
P_{-+}^{(k)}(\omega) & P_{++}^{(k)}(\omega)
\end{array}\right), \quad \text { (hereafter, } k \geq 1\right),
\end{array}
$$

where the elements of diagonalization martix $U_{\omega}$ are combinations of the LO anomalous dimensions; i.e.,

$$
U_{\omega}=\left(\begin{array}{cc}
1 & -1 \\
\frac{1-\alpha_{\omega}^{(0)}}{\varepsilon_{\omega}^{(0)}} & \frac{\alpha_{\omega}^{(0)}}{\varepsilon_{\omega}^{(0)}}
\end{array}\right), \quad U_{\omega}^{-1}=\left(\begin{array}{ll}
\alpha_{\omega}^{(0)} & \varepsilon_{\omega}^{(0)} \\
\alpha_{\omega}^{(0)}-1 & \varepsilon_{\omega}^{(0)}
\end{array}\right),
$$

with

$$
\alpha_{\omega}^{(0)}=\frac{P_{q q}^{(0)}(\omega)-P_{++}^{(0)}(\omega)}{P_{--}^{(0)}(\omega)-P_{++}^{(0)}(\omega)}, \quad \epsilon_{\omega}^{(0)}=\frac{P_{g q}^{(0)}(\omega)}{P_{--}^{(0)}(\omega)-P_{++}^{(0)}(\omega)},
$$

and

$$
P_{ \pm \pm}^{(0)}(\omega)=\frac{1}{2}\left[P_{q q}^{(0)}(\omega)+P_{g g}^{(0)}(\omega) \pm \sqrt{\left(P_{q q}^{(0)}(\omega)-P_{g g}^{(0)}(\omega)\right)^{2}+4 P_{q g}^{(0)}(\omega) P_{g q}^{(0)}(\omega)}\right]
$$

The components $P_{--}^{(k)}(\omega)(k \geq 1)$ of the timelike-splitting-function matrix can be obtained as [39]

$$
\begin{aligned}
& P_{--}^{(k)}(\omega)=\alpha_{\omega}^{(0)} P_{q q}^{(k)}(\omega)+\epsilon_{\omega}^{(0)} P_{q g}^{(k)}(\omega)+\beta_{\omega}^{(0)} P_{g q}^{(k)}(\omega)+\left(1-\alpha_{\omega}^{(0)}\right) P_{g g}^{(k)}(\omega), \\
& P_{-+}^{(k)}(\omega)=P_{--}^{(k)}(\omega)-\left(P_{q q}^{(k)}(\omega)+\frac{1-\alpha_{\omega}^{(0)}}{\epsilon_{\omega}^{(0)}} P_{g q}^{(k)}(\omega)\right), \\
& P_{++}^{(k)}(\omega)=P_{q q}^{(k)}(\omega)+P_{g g}^{(k)}(\omega)-P_{--}^{(k)}(\omega), \\
& P_{+-}^{(k)}(\omega)=P_{++}^{(k)}(\omega)-\left(P_{q q}^{(k)}(\omega)-\frac{\alpha_{\omega}^{(0)}}{\epsilon_{\omega}^{(0)}} P_{g q}^{(k)}(\omega)\right)=P_{g g}^{(k)}(\omega)-\left(P_{--}^{(k)}(\omega)-\frac{\alpha_{\omega}^{(0)}}{\epsilon_{\omega}^{(0)}} P_{g q}^{(k)}(\omega)\right),
\end{aligned}
$$


where

$$
\beta_{\omega}^{(0)}=\frac{P_{q g}^{(0)}(\omega)}{P_{--}^{(0)}(\omega)-P_{++}^{(0)}(\omega)}=\frac{\alpha_{\omega}^{(0)}\left(1-\alpha_{\omega}^{(0)}\right)}{\epsilon_{\omega}^{(0)}} .
$$

The corresponding \pm components of the fragmentation functions have the following form:

$$
\begin{aligned}
\left(\begin{array}{c}
D_{-}(\omega) \\
D_{+}(\omega)
\end{array}\right) & =U_{\omega}^{-1}\left(\begin{array}{c}
D_{s}(\omega) \\
D_{g}(\omega)
\end{array}\right) \\
& =\left(\begin{array}{c}
\left(\alpha_{\omega}^{(0)} D_{s}(\omega)+\varepsilon_{\omega}^{(0)} D_{g}(\omega)\right. \\
\left.\alpha_{\omega}^{(0)}-1\right) D_{s}(\omega)+\varepsilon_{\omega}^{(0)} D_{g}(\omega)
\end{array}\right) .
\end{aligned}
$$

As it was mentioned above, the considered diagonalization gives a possibility to analyze FFs themselves. When we study the multiplicities, we should take into account the small $x$ resummation, which corresponds to the resummation near $N=1$ in the momentum space. It is quite easy to perform this resummation for the diagonal elements $P_{ \pm \pm}^{(k)}(\omega)$ but rather difficult in the case of the nondiagonal ones $P_{ \pm \mp}^{(k)}(\omega)$ (see discussion is Ref. [7]).

\section{A. Direct diagonalization of parton multiplicities}

In the important simplification of QCD, namely $\mathcal{N}=4$ SYM, a diagonalization was performed $[40,41]$ at all orders of perturbation theory, where the corresponding matrix contains the respective combinations of anomalous dimensions. Technically, it corresponds to the replacement $P_{a, b}^{(0)}(\omega) \rightarrow P_{a, b}\left(\omega, a_{s}\right)$ as in (1.6). ${ }^{5}$

Following to this case, in Ref. [3], a similar diagonalization was applied to the case of multiplicities. So, we solved Eq. (1.3) for $\omega=0$ exactly by exploiting Eqs. (2.32) and (2.35). To this end, we diagonalize the NNLL DGLAP evolution kernel as

$$
\begin{array}{ll}
U^{-1}\left(\begin{array}{ll}
P_{q q} & P_{g q} \\
P_{q g} & P_{g g}
\end{array}\right) & U=\left(\begin{array}{ll}
P_{--} & 0 \\
0 & P_{++}
\end{array}\right), \\
U=\left(\begin{array}{ll}
1 & -1 \\
\frac{1-\alpha}{\varepsilon} & \frac{\alpha}{\varepsilon}
\end{array}\right), & U^{-1}=\left(\begin{array}{ll}
\alpha & \varepsilon \\
\alpha-1 & \varepsilon
\end{array}\right),
\end{array}
$$

where

\footnotetext{
${ }^{5}$ Strictly speaking, such replacement is directly applicable in the polarized case, while in spin-averaged case $(a, b=q, g, \varphi)$, since the contributions from scalars should be added.
}

$$
\begin{aligned}
\alpha & =\frac{P_{q q}-P_{++}}{P_{--}-P_{++}}, \quad \varepsilon=\frac{P_{g q}}{P_{--}-P_{++}}, \\
P_{ \pm \pm} & =\frac{1}{2}\left[P_{q q}+P_{g g} \pm \sqrt{\left(P_{q q}-P_{g g}\right)^{2}+4 P_{q g} P_{g q}}\right]
\end{aligned}
$$

where $P_{a, b}$ are given in Eqs. (2.32) and (2.33).

Acting by the operator $U^{-1}$ to Eq. (1.3) for $N=1$ from the left (i.e., multiplying by the respective matrix), we rewrite it as

$$
U^{-1} \frac{\mu^{2} d}{d \mu^{2}}\left[U U^{-1}\left(\begin{array}{c}
D_{s} \\
D_{g}
\end{array}\right)\right]=U^{-1}\left(\begin{array}{cc}
P_{q q} & P_{g q} \\
P_{q g} & P_{g g}
\end{array}\right) U U^{-1}\left(\begin{array}{c}
D_{s} \\
D_{g}
\end{array}\right)
$$

Using the \pm components of multiplicities as

$$
\left(\begin{array}{l}
D_{-} \\
D_{+}
\end{array}\right)=U^{-1}\left(\begin{array}{c}
D_{s} \\
D_{g}
\end{array}\right)=\left(\begin{array}{l}
\left(\alpha D_{s}+\varepsilon D_{g}\right. \\
\alpha-1) D_{s}+\varepsilon D_{g}
\end{array}\right)
$$

we rewrite the above Eq. (3.10) as

$U^{-1} \frac{\mu^{2} d}{d \mu^{2}}\left[U\left(\begin{array}{l}D_{-} \\ D_{+}\end{array}\right)\right]=\left(\begin{array}{ll}P_{--} & 0 \\ 0 & P_{++}\end{array}\right)\left(\begin{array}{l}D_{-} \\ D_{+}\end{array}\right)$.

The essential difference between QCD and $\mathcal{N}=4 \mathrm{SYM}$ is the $\mu^{2}$ dependence of the strong coupling constant, which propagates, in turn, to the matrix $U$. So, now the matrix $U$ and the operator $\mu^{2} \frac{d}{d \mu^{2}}$ do not commute. To have a usual form of diagonal DGLAP equations, we can rewrite Eq. (3.12) in the form,

$$
\begin{aligned}
& \mu^{2} \frac{d}{d \mu^{2}}\left(\begin{array}{l}
D_{-} \\
D_{+}
\end{array}\right) \\
& \quad=\left[\left(\begin{array}{ll}
P_{--} & 0 \\
0 & P_{++}
\end{array}\right)-U^{-1}\left(\mu^{2} \frac{d}{d \mu^{2}}\right) U\right]\left(\begin{array}{c}
D_{-} \\
D_{+}
\end{array}\right),
\end{aligned}
$$

where the second term contained within the square brackets stems from the commutator of $\mu^{2} \frac{d}{d \mu^{2}}$ and $U$.

Owing to Eq. (2.35), the square root in Eq. (3.9) is exactly canceled, and we have simple expressions for $P_{ \pm \pm}$,

$$
\begin{gathered}
P_{--}=-A, \quad P_{++}=P_{q q}+P_{g g}+A, \\
\alpha=\frac{P_{g g}+A}{P_{q q}+P_{g g}+2 A}, \quad \varepsilon=-C \alpha .
\end{gathered}
$$

Let us stress, as it was already mentioned at the end of the previous section, that the presence of resummation of anomalous dimensions and direct diagonalization of parton multiplicities leads to a strong simplification of the values 
of diagonal anomalous dimensions, in which all square roots have shrunk, which, so far, has only been observed in supersymmetric generalizations of QCD.

Inserting Eq. (3.14) in Eq. (3.8), we have

$$
U^{-1}\left(\mu^{2} \frac{d}{d \mu^{2}}\right) U=-\frac{1}{\alpha}\left(\mu^{2} \frac{d}{d \mu^{2}}\right) \alpha\left(\begin{array}{ll}
1 & 0 \\
1 & 0
\end{array}\right)
$$

Using the QCD $\beta$ function,

$$
\begin{aligned}
\mu^{2} \frac{d}{d \mu^{2}} a_{s} & =\beta\left(a_{s}\right)=-\beta_{0} a_{s}^{2}-\beta_{1} a_{s}^{3}+\mathcal{O}\left(a_{s}^{4}\right), \\
\beta_{0} & =\frac{C_{A}}{3}(11-2 \varphi), \\
\beta_{1} & =\frac{2 C_{A}^{2}}{3}[17-\varphi(5+3 C)],
\end{aligned}
$$

after a small algebra, we may cast Eq. (1.3) in its final form,

$\mu^{2} \frac{d}{d \mu^{2}}\left(\begin{array}{l}D_{-} \\ D_{+}\end{array}\right)=\left(\begin{array}{ll}\frac{C \varphi \beta_{0}}{3 C_{A}} \gamma_{0}^{3}-A & 0 \\ \frac{C \varphi \beta_{0}}{3 C_{A}} \gamma_{0}^{3} & P_{g g}+P_{q q}+A\end{array}\right)\left(\begin{array}{c}D_{-} \\ D_{+}\end{array}\right)$.

The initial conditions are given by Eq. (3.11) for $\mu=\mu_{0}$ in terms of the three constants $\alpha_{s}\left(\mu_{0}^{2}\right), D_{s}\left(\mu_{0}^{2}\right)$, and $D_{g}\left(\mu_{0}^{2}\right)$.

\section{B. Results}

As seen from the Eq. (3.17), the "-" component $D_{-}$can be obtained as the general solution of a homogeneous differential equation. It has the following form [3]:

$$
\begin{aligned}
\frac{D_{-}\left(\mu^{2}\right)}{D_{-}\left(\mu_{0}^{2}\right)} & =\exp \left[\int_{\mu_{0}^{2}}^{\mu^{2}} \frac{d \bar{\mu}^{2}}{\bar{\mu}^{2}}\left(\frac{C \varphi \beta_{0}}{3 C_{A}} \gamma_{0}^{3}\left(\bar{\mu}^{2}\right)-A\left(\bar{\mu}^{2}\right)\right)\right] \\
& =\frac{T_{-}\left(\gamma_{0}\left(\mu^{2}\right)\right)}{T_{-}\left(\gamma_{0}\left(\mu_{0}^{2}\right)\right)},
\end{aligned}
$$

where

$$
T_{-}\left(\gamma_{0}\right)=\gamma_{0}^{d_{-}} \exp \left(-\frac{4}{3} C \varphi \gamma_{0}\right), \quad d_{-}=\frac{8 C_{A}}{3 \beta_{0}} C \varphi .
$$

The "+" component $D_{+}$obeys [3] to the inhomogeneous differential equation. The general solution $\tilde{D}_{+}$of its homogeneous part is

$$
\begin{aligned}
\frac{\tilde{D}_{+}\left(\mu^{2}\right)}{\tilde{D}_{+}\left(\mu_{0}^{2}\right)}= & \exp \left[\int _ { \mu _ { 0 } ^ { 2 } } ^ { \mu ^ { 2 } } \frac { d \overline { \mu } ^ { 2 } } { \overline { \mu } ^ { 2 } } \gamma _ { 0 } ( \overline { \mu } ^ { 2 } ) \left(1+\left(2 K_{q}^{(1)}+K_{g}^{(1)}\right) \gamma_{0}\left(\bar{\mu}^{2}\right)\right.\right. \\
& \left.\left.+\left(K_{q}^{(2)}+K_{g}^{(2)}\right) \gamma_{0}^{2}\left(\bar{\mu}^{2}\right)\right)\right] \\
= & \frac{T_{+}\left(\gamma_{0}\left(\mu^{2}\right)\right)}{T_{+}\left(\gamma_{0}\left(\mu_{0}^{2}\right)\right)}
\end{aligned}
$$

where

$$
\begin{aligned}
T_{+}\left(\gamma_{0}\right) & =\gamma_{0}^{d_{+}} \exp \left[\frac{4 C_{A}}{\beta_{0} \gamma_{0}}-\frac{4 C_{A}}{\beta_{0}}\left(K_{q}^{(2)}+K_{g}^{(2)}-b_{1}\right) \gamma_{0}\right], \\
d_{+} & =-\frac{4 C_{A}}{\beta_{0}}\left(2 K_{q}^{(1)}+K_{g}^{(1)}\right),
\end{aligned}
$$

and $b_{1}=\beta_{1} /\left(2 C_{A} \beta_{0}\right)$.

Adding to $\tilde{D}_{+}$a special solution of the inhomogeneous differential equation for $D_{+}$, we find its general solution [3]:

$$
\begin{aligned}
D_{+}\left(\mu^{2}\right)= & {\left[\frac{D_{+}\left(\mu_{0}^{2}\right)}{T_{+}\left(\gamma_{0}\left(\mu_{0}^{2}\right)\right)}-\frac{4}{3} C \varphi \frac{D_{-}\left(\mu_{0}^{2}\right)}{T_{-}\left(\gamma_{0}\left(\mu_{0}^{2}\right)\right)}\right.} \\
& \left.\times \int_{\gamma_{0}\left(\mu_{0}^{2}\right)}^{\gamma_{0}\left(\mu^{2}\right)} \frac{d \bar{\gamma}_{0}}{1+b_{1} \bar{\gamma}_{0}^{2}} \frac{T_{-}\left(\bar{\gamma}_{0}\right)}{T_{+}\left(\bar{\gamma}_{0}\right)}\right] T_{+}\left(\gamma_{0}\left(\mu^{2}\right)\right) .
\end{aligned}
$$

\section{CASIMIR SCALING}

In the supersymmetric generalization of QCD (SQCD), the corresponding relation [i.e., (2.35) with $C=1$ ] exists $[2,20,22]$ for the anomalous dimensions $P_{a b}^{\mathrm{SUSY}}(N)$ with arbitrary $N$ values $^{6}$ :

$$
P_{q q}^{\mathrm{SUSY}}(x)+P_{g q}^{\mathrm{SUSY}}(x)=P_{g g}^{\mathrm{SUSY}}(x)+P_{q g}^{\mathrm{SUSY}}(x) .
$$

Beyond LO, the property (4.1) is violated in the standard dimension regularization, but it survives in the form of the dimensional reduction [42] and was used also to check real calculations (see Ref. [43,44] and discussion therein). It seems that the relation (4.1) is violated [6] at the NNLO level of accuracy, but this requires some additional investigation.

It will be interesting to check whether Eq. (2.35) also holds beyond $\mathcal{O}\left(\gamma_{0}^{3}\right)$ in the case of the dimensional reduction [42]. The choice of a scheme in the above consideration is not so important because a difference in the results of various schemes is exactly canceled in Eq. (2.35).

Following [45], Eq. (4.1) can be spelled out as an equality of the total probabilities of quark and gluon

\footnotetext{
${ }^{6}$ In fact, it was observed for the splitting functions $P_{a b}^{\mathrm{SUSY}}(x)$, which correspond to the $P_{a b}^{\mathrm{SUSY}}(N)$ [see Eq. (1.5)].
} 
decays. We note that such probabilistic interpretation becomes to be very important directly in QCD [46,47] for decoupling of orbital and total angular momenta in the nucleon.

Following [45,46], we can explore the probabilistic properties hidden in Eq. (2.35). To do it, we introduce a new form of the quark $\bar{D}_{s}$ and gluon $\bar{D}_{g}$ multiplicities,

$$
D_{s}\left(\mu^{2}\right)=C_{F} \bar{D}_{s}\left(\mu^{2}\right), \quad D_{g}\left(\mu^{2}\right)=C_{A} \bar{D}_{g}\left(\mu^{2}\right),
$$

where we extract the corresponding color charges $C_{F}$ and $C_{A}$, respectively.

The new multiplicities obey the following DGLAP equations:

$$
\mu^{2} \frac{d}{d \mu^{2}}\left(\begin{array}{c}
\bar{D}_{s}\left(\mu^{2}\right) \\
\bar{D}_{g}\left(\mu^{2}\right)
\end{array}\right)=\left(\begin{array}{cc}
\bar{P}_{q q} & \bar{P}_{g q} \\
\bar{P}_{q g} & \bar{P}_{g g}
\end{array}\right)\left(\begin{array}{c}
\bar{D}_{s}\left(\mu^{2}\right) \\
\bar{D}_{g}\left(\mu^{2}\right)
\end{array}\right),
$$

where

$$
\bar{P}_{a a}=P_{a a}, \quad \bar{P}_{q g}=C P_{q g}, \quad \bar{P}_{g q}=C^{-1} P_{q q},
$$

and the relation (2.35) becomes to be as follows:

$$
\bar{P}_{q q}+\bar{P}_{g q}=\bar{P}_{g g}+\bar{P}_{q g} ;
$$

i.e., it is exactly equal (for $N=1$ ) to the one in (4.1) obtained in the SQCD framework.

So, the new parton multiplicities $\bar{D}_{a}$ have the same probabilistic properties as the original ones $D_{a}$ in the supersymmetric case, bringing it closer to observable quantities.

Since the parton multiplicities $\bar{D}_{a}$ are proportional to the standard ones $D_{a}$, the solution of the DGLAP equation (4.3) is the same as one done in Ref. [3] for Eq. (1.3) at $N=1$ : after diagonalization of (4.3), there are two solutions in the form of so-called "+" and "-" components.

\section{A. High-energy asymptotics of multiplicities}

Returning to the parton basis, it is useful to decompose $\bar{D}_{a}=\bar{D}_{a}^{+}+\bar{D}_{a}^{-}$into the large and small components $\bar{D}_{a}^{ \pm}$ proportional to $D_{ \pm}$, respectively. Defining $\bar{r}_{ \pm}=\bar{D}_{g}^{ \pm} / \bar{D}_{s}^{ \pm}$ and using Eqs. (2.32), (2.33), and (3.20), we then have $C_{F} \bar{D}_{s}^{ \pm}=\mp D_{ \pm}$and

$\bar{r}_{+}=1+\mathcal{O}\left(\gamma_{0}^{2}\right)$,

$\bar{r}_{-}=-\frac{4}{3} n_{f} \gamma_{0}+\frac{n_{f}}{18}[29-2 \varphi(5-2 C)] \gamma_{0}^{2}+\mathcal{O}\left(\gamma_{0}^{3}\right)$.

Recalling that $\bar{r}=\bar{D}_{g} / \bar{D}_{s}$, we have

$$
\bar{r}=\frac{\bar{r}_{+}+\bar{r}_{-} \bar{D}_{s}^{-} / \bar{D}_{s}^{+}}{1+\bar{D}_{s}^{-} / \bar{D}_{s}^{+}},
$$

So, for the high-energy asymptotics (i.e., $\mu \rightarrow \infty$ ), where the " + " component strongly dominates, we have for the ratio $\bar{r}$ :

$$
\bar{r} \rightarrow \bar{r}_{+}=1 ;
$$

i.e., the new multiplicities of gluon and quark jets become equal in all known orders. This equality corresponds exactly to the Casimir scaling (i.e., to $D_{g}^{+} / D_{s}^{+}=$ $\left.C_{A} / C_{F}\right)$ mentioned above. One should expect that this equality should be clearly seen experimentally and thus, can be regarded as a guideline in high-energy experiments and a complementary tool for discrimination of quark and gluon jets.

When going to lower-energy values, this equivalence should be violated. One of the important elements of the violation is the appearance of contributions proportional to the quartic Casimirs in high orders of the perturbation theory (see investigations in Refs. [10,48-51]). So, we think that the equality $\bar{r}_{+}=1$ may be kept up to $a_{s}^{4} \sim \gamma_{0}^{8}$ accuracy, ${ }^{7}$ where the corresponding splitting functions $P_{b a}$ would contain Feynman diagrams coming with the quartic Casimir contributions.

However, this is not the only source of violation of the property $\bar{r}=1$. As seen from Eq. (4.7), the existence of the "-" component violates the equality (4.8) between the new multiplicities $\bar{D}_{s}$ and $\bar{D}_{g}$ that may be essentially stronger than the possible violation due the quartic Casimir contributions. Of course, the "-" component does not increase with energy increasing, but its contribution leads to a nontrivial dependence of the ratio of gluon and quark multiplicities, which is important at nonasymptotically large energy values. Such nontrivial dependence is seen in experimental data (see Refs. [3,7,52] and discussions therein). We note that the ratio $\bar{r}_{-} \sim n_{f}$ and, thus, the equality (4.8), should be violated in pure gluodynamics, essentially slowly, i.e., at $a_{s}^{4} \sim \gamma_{0}^{8}$ accuracy by contributions of the quartic Casimirs.

We note also that the contribution of the "-" component is very important $[3,7,52]$ for comparison of the theoretical predictions for the parton jet multiplicities with the experimental data, which belongs to the subasymptotic range. Indeed, as it was shown in [7,52], the "-" component contribution gives the natural explanation of the longstanding discrepancy in the theoretical description of the data, which was reviewed, for example, in Ref. [53]. In a sense, the presence of the "-" component leads to a rather different evolution of the gluon and quark multiplicities, which is in good agreement with experimental data.

The importance of the "-" component contribution is also in full agreement with the study [54] of low $x$

\footnotetext{
${ }^{7}$ Note that the quartic Casimir contributions may be negligible numerically [48], and the "Casimir scaling" may be fulfilled even above $a_{s}^{4} \sim \gamma_{0}^{8}$ accuracy in approximated form.
} 
asymptotics of parton densities, where the existence of the corresponding "-" component leads to a good agreement between theoretical studies [55] and the experimental data [56] for the structure function $F_{2}\left(x, Q^{2}\right)$ of the deepinelastic scattering obtained by the $\mathrm{H} 1$ and ZEUS Collaborations.

\section{CONCLUSIONS}

In this article, we study in some detail the SUSY-like relation [3] between the NNLL-resummed first Mellin moments of the timelike DGLAP splitting functions in real QCD, in Eq. (2.35). This relation appeared through the small- $x$ resummation of the timelike splitting functions and nonstandard diagonalization [3] of their first Mellin moments. In Secs. II and III, we presented the basic steps of the resummation and the diagonalization, respectively.

In Eq. (4.2), we introduced the new quark and gluon jet multiplicities $\bar{D}_{a}$, which have probabilistic properties, the same as for the standard multiplicities $D_{a}$ in the framework of the supersymmetric extension of QCD. As it was already discussed in Sec. IVA, the new quark and gluon multiplicities $\bar{D}_{a}$ should have similar behavior at high energies that can be regarded as a guideline in high-energy experiments.
The violation of this similarity in the region of lower energies is controlled by the violation of the Casimir scaling, both due to the appearance of contributions proportional to the quartic Casimirs and due to the "-" component. At high energies, these effects are small, which is associated with the fact that the contributions proportional to the quartic Casimirs are suppressed by $\sim \alpha_{s}^{2}$ and the "-" component does not contain the factor $\sim \exp \left[\sim 1 / \sqrt{\alpha_{s}}\right]$ growing in the high-energy region, and thus, is also strongly suppressed. With decreasing energy, these effects will begin to manifest themselves, leading to different energy dependence of quark and gluon multiplicities.

Such a similarity of parton multiplicities $\bar{D}_{a}$ at high energies and the appearance of differences at lower energies can be studied in experiments performed at LHC and at a future Electron-Ion Collider.

\section{ACKNOWLEDGMENTS}

A. V. K. thanks Prof. Bernd Kniehl for the joint work during the preparation of Ref. [3], which is the base for current investigations. The work of O. V. T. was partially supported by RFBR Grant No. 18-02-01107.
[1] V. N. Gribov and L. N. Lipatov, Sov. J. Nucl. Phys. 15, 438 (1972); G. Altarelli and G. Parisi, Nucl. Phys. B126, 298 (1977).

[2] Yu. L. Dokshitzer, Sov. Phys. JETP 46, 641 (1977).

[3] B. A. Kniehl and A. V. Kotikov, arXiv:1702.03193.

[4] M. Gluck, E. Reya, and A. Vogt, Phys. Rev. D 48, 116 (1993); 51, 1427(E) (1995).

[5] S. Moch and A. Vogt, Phys. Lett. B 659, 290 (2008).

[6] A. A. Almasy, S. Moch, and A. Vogt, Nucl. Phys. B854, 133 (2012).

[7] P. Bolzoni, B. A. Kniehl, and A. V. Kotikov, Nucl. Phys. B875, 18 (2013).

[8] S. J. Brodsky and J. F. Gunion, Phys. Rev. Lett. 37, 402 (1976); K. Konishi, A. Ukawa, and G. Veneziano, Phys. Lett. 78B, 243 (1978).

[9] P. Cvitanovic, Phys. Rev. D 14, 1536 (1976).

[10] C. Anzai, Y. Kiyo, and Y. Sumino, Nucl. Phys. B838, 28 (2010); A. Grozin, J. Henn, and M. Stahlhofen, J. High Energy Phys. 10 (2017) 052.

[11] G. P. Korchemsky, Mod. Phys. Lett. A 04, 1257 (1989).

[12] T. Becher and M. Neubert, Phys. Rev. Lett. 102, 162001 (2009); J. High Energy Phys. 06 (2009) 081; Phys. Rev. D 79, 125004 (2009); S. Moch, J. A. M. Vermaseren, and A. Vogt, Nucl. Phys. B688, 101 (2004).

[13] E. Gardi and L. Magnea, J. High Energy Phys. 03 (2009) 079.

[14] L. J. Dixon, Phys. Rev. D 79, 091501 (2009).
[15] A. Ferroglia, M. Neubert, B. D. Pecjak, and L. L. Yang, Phys. Rev. Lett. 103, 201601 (2009); J. High Energy Phys. 11 (2009) 062.

[16] T. Becher and M. Neubert, Eur. Phys. J. C 71, 1665 (2011); M. G. Echevarria, I. Scimemi, and A. Vladimirov, Phys. Rev. D 93, 054004 (2016).

[17] C. W. Bernard, Nucl. Phys. B219, 341 (1983); J. Ambjorn, P. Olesen, and C. Peterson, Nucl. Phys. B240, 189 (1984).

[18] A. H. Mueller, Phys. Lett. 104B, 161 (1981).

[19] A. Vogt, J. High Energy Phys. 10 (2011) 025.

[20] C. H. Kom, A. Vogt, and K. Yeats, J. High Energy Phys. 10 (2012) 033.

[21] B. A. Kniehl and A. V. Kotikov, Phys. Part. Nucl. 49, 921 (2018); A. Kotikov, EPJ Web Conf. 191, 04006 (2018); Phys. Part. Nucl. Lett. 16, 427 (2019).

[22] C. Kounnas and D. A. Ross, Nucl. Phys. B214, 317 (1983); A. P. Bukhvostov, G. V. Frolov, L. N. Lipatov, and E. A. Kuraev, Nucl. Phys. B258, 601 (1985); Yu. L. Dokshitzer, V. A. Khoze, A. H. Mueller, and S. I. Troian, Basics of Perturbative QCD (Editions Frontières, Gif-sur-Yvette, 1991).

[23] S. Albino, P. Bolzoni, B. A. Kniehl, and A. Kotikov, Nucl. Phys. B851, 86 (2011); B855, 801 (2012).

[24] A. H. Mueller, Nucl. Phys. B213, 85 (1983).

[25] P. J. Rijken and W. L. van Neerven, Phys. Lett. B 386, 422 (1996); Nucl. Phys. B487, 233 (1997); P. J. Rijken and W. L. van Neerven, Phys. Lett. B 392, 207 (1997). 
[26] A. Mitov, S. Moch, and A. Vogt, Phys. Lett. B 638, 61 (2006); A. Mitov and S. O. Moch, Nucl. Phys. B751, 18 (2006).

[27] J. Blumlein and V. Ravindran, Nucl. Phys. B749, 1 (2006).

[28] S. Albino, P. Bolzoni, B. A. Kniehl, and A. Kotikov, arXiv: 1107.1142.

[29] A. Bassetto, M. Ciafaloni, G. Marchesini, and A. H. Mueller, Nucl. Phys. B207, 189 (1982).

[30] A. Bassetto, M. Ciafaloni, and G. Marchesini, Phys. Rep. 100, 201 (1983).

[31] G. Curci, W. Furmanski, and R. Petronzio, Nucl. Phys. B175, 27 (1980).

[32] S. Catani and F. Hautmann, Nucl. Phys. B427, 475 (1994).

[33] R. K. Ellis, H. Georgi, M. Machacek, H. D. Politzer, and G. G. Ross, Nucl. Phys. B152, 285 (1979).

[34] A. Vogt, C. H. Kom, N. A. Lo Presti, G. Soar, A. A. Almasy, S. Moch, J. A. M. Vermaseren, and K. Yeats, Proc. Sci., LL2012 (2012) 004 [arXiv:1212.2932].

[35] J. Fleischer, A. V. Kotikov, and O. L. Veretin, Nucl. Phys. B547, 343 (1999).

[36] A. V. Kotikov, L. N. Lipatov, A. Rej, M. Staudacher, and V. N. Velizhanin, J. Stat. Mech. (2007) P10003.

[37] A. V. Kotikov, A. Rej, and S. Zieme, Nucl. Phys. B813, 460 (2009); M. Beccaria, A. V. Belitsky, A. V. Kotikov, and S. Zieme, Nucl. Phys. B827, 565 (2010).

[38] C. Marboe and V. Velizhanin, J. High Energy Phys. 11 (2016) 013.

[39] A. J. Buras, Rev. Mod. Phys. 52, 199 (1980).

[40] A. V. Kotikov and L. N. Lipatov, Nucl. Phys. B661, 19 (2003); B685, 405(E) (2004).

[41] L. Bianchi, V. Forini, and A. V. Kotikov, Phys. Lett. B 725, 394 (2013).

[42] W. Siegel, Phys. Lett. 84B, 193 (1979).

[43] R. Mertig and W. L. van Neerven, Z. Phys. C 70, 637 (1996).

[44] I. Antoniadis and E. G. Floratos, Nucl. Phys. B191, 217 (1981); A. P. Bukhvostov, E. A. Kuraev, L. N. Lipatov, and
G. V. Frolov, JETP Lett. 41, 92 (1985); M. Stratmann and W. Vogelsang, Nucl. Phys. B496, 41 (1997).

[45] Y. L. Dokshitzer, Phys. At. Nucl. 71, 636 (2008).

[46] O. V. Teryaev, arXiv:hep-ph/9803403.

[47] X. Artru, M. Elchikh, J. M. Richard, J. Soffer, and O. V. Teryaev, Phys. Rep. 470, 1 (2009).

[48] S. Catani, D. De Florian, and M. Grazzini, Eur. Phys. J. C 79, 685 (2019); S. Moch, B. Ruijl, T. Ueda, J. A. M. Vermaseren, and A. Vogt, Phys. Lett. B 782, 627 (2018).

[49] R. N. Lee, A. V. Smirnov, V. A. Smirnov, and M. Steinhauser, Phys. Rev. D 94, 054029 (2016); P. Arnold, Phys. Rev. D 99, 054017 (2019); S. Catani, D. Colferai, and A. Torrini, J. High Energy Phys. 01 (2020) 118; T. Becher and M. Neubert, J. High Energy Phys. 01 (2020) 025.

[50] J. M. Henn, T. Peraro, M. Stahlhofen, and P. Wasser, Phys. Rev. Lett. 122, 201602 (2019); J. M. Henn, G. P. Korchemsky, and B. Mistlberger, J. High Energy Phys. 04 (2020) 018; A. von Manteuffel and R. M. Schabinger, Phys. Rev. D 99, 094014 (2019); T. Huber, A. von Manteuffel, E. Panzer, R. M. Schabinger, and G. Yang, Phys. Lett. B 807, 135543 (2020); A. von Manteuffel, E. Panzer, and R. M. Schabinger, Phys. Rev. Lett. 124, 162001 (2020).

[51] G. Das, S. O. Moch, and A. Vogt, J. High Energy Phys. 03 (2020) 116.

[52] P. Bolzoni, B. A. Kniehl, and A. V. Kotikov, Phys. Rev. Lett. 109, 242002 (2012).

[53] I. M. Dremin and J. W. Gary, Phys. Rep. 349, 301 (2001); I. M. Dremin, Phys. Usp. 45, 507 (2002).

[54] A. V. Kotikov and G. Parente, Nucl. Phys. B549, 242 (1999); G. Cvetič, A. Yu. Illarionov, B. A. Kniehl, and A. V. Kotikov, Phys. Lett. B 679, 350 (2009).

[55] A. V. Kotikov and B. G. Shaikhatdenov, Phys. Part. Nucl. 44, 543 (2013); Phys. At. Nucl. 78, 525 (2015); Phys. Part. Nucl. 48, 829 (2017).

[56] F. D. Aaron et al. (H1 and ZEUS Collaborations), J. High Energy Phys. 01 (2010) 109. 